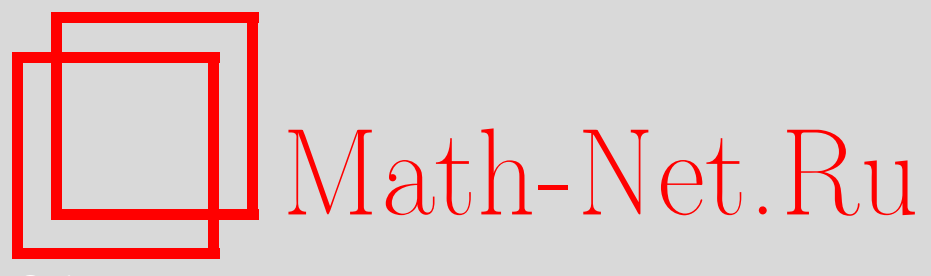

А. П. Старовойтов, Н. А. Старовойтова, Об асимптотике строк таблицы Паде аналитических функций с логарифмическими точками ветвления, Матем. заметки, 2008, том 84, выпуск 3, 409-419

DOI: https://doi.org/10.4213/mzm4049

Использование Общероссийского математического портала Math-Net.Ru подразумевает, что вы прочитали и согласны с пользовательским соглашением http: //www . mathnet.ru/rus/agreement

Параметры загрузки:

IP : 3.81 .55 .215

26 апреля 2023 г., 09:39:58

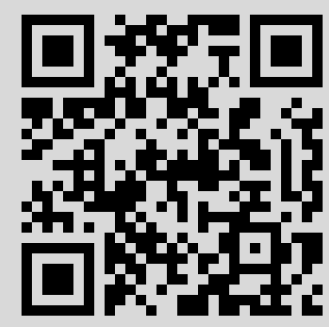


Том 84 выпуск 3 сентябрь 2008

\section{Об асимптотике строк таблицы Паде аналитических функций с логарифмическими точками ветвления}

\section{А. П. Старовойтов, Н. А. Старовойтова}

Для функций $f(z)=\sum_{n=0}^{\infty} z^{l_{n}} / a_{n}$, где $l_{n}$ и $a_{n}$ - арифметические прогрессии, и их аппроксимаций Паде $\pi_{n, m}(z ; f)$ установлена асимптотика убывания разности $f(z)-\pi_{n, m}(z ; f)$ в случае, когда $z \in D=\{z:|z|<1\}, m$ фиксировано, а $n \rightarrow \infty$. В частности, найдены точные порядки убывания наилучших равномерных рациональных приближений функций $\ln (1-z), \operatorname{arctg} z$ в круге $D_{q}=\{z:|z| \leqslant q<1\}$.

Библиография: 20 названий.

1. Введение. Пусть $\mathscr{A}(b)=\mathscr{A}\left(b, a_{0}\right)$ - множество аналитических в $D=\{z$ : $|z|<1\}$ функций $f$, представимых в виде

$$
f(z)= \pm \sum_{n=0}^{\infty} \frac{z^{n}}{a_{n}}
$$

где $a_{n}=b n+a_{0}-$ арифметическая прогрессия и $a_{n} \neq 0$. Для простоты в дальнейшем ограничимся случаем, когда $b$ и $a_{0}$ - действительные числа, причем $b>0, a_{0}>0$. По определению полагаем

$$
\mathscr{A}^{-}(b)=\{f(-z): f \in \mathscr{A}(b)\} .
$$

Если $l_{n}=d n+l_{0}$, где $d \in \mathbb{N}, l_{0} \in \mathbb{N} \cup\{0\}$ - фиксированные числа, то будем говорить, что функция $g \in \mathscr{A}(b ; d)=\mathscr{A}\left(b, a_{0} ; d, l_{0}\right)$ тогда и только тогда, когда она представима в виде $g(z)=z^{l_{0}} f\left(z^{d}\right)$, где $f \in \mathscr{A}(b)$. Аналогично по определению полагаем

$$
\mathscr{A}^{-}(b ; d)=\left\{g(z): g(z)=z^{l_{0}} f\left(-z^{d}\right), f \in \mathscr{A}(b)\right\} .
$$

Так, например, функция

$$
\ln (1-z)=-\sum_{n=1}^{\infty} \frac{z^{n}}{n}
$$

принадлежит $\mathscr{A}(1,0)$, а

$$
\operatorname{arctg} z=\sum_{n=0}^{\infty} \frac{(-1)^{n}}{2 n+1} z^{2 n+1}
$$

является функцией из класса $\mathscr{A}^{-}(2,1 ; 2,1)$. Заметим, что на границе круга сходимости $\ln (1-z)$ и $\operatorname{arctg} z$ имеют соответственно особые точки $1, \pm i$, которые являются логарифмическими точками ветвления этих функций.

(C) А. П. Старовойтов, Н. А. Старовойтова, 2008 
Anпроксимачией Паде $\pi_{n, m}(z ; f)=p_{n}(z ; f) / q_{m}(z ; f)$ степенного ряда (1) назовем аналитическую в нуле рациональную функцию (р.ф.) вида

$$
r_{n, m}(z)=\frac{a_{0}+a_{1} z+\cdots+a_{n} z^{n}}{b_{0}+b_{1} z+\cdots+a_{m} z^{m}},
$$

имеющую максимально возможный порядок касания к ряду (1), т.е. такую, для которой в некоторой окрестности нуля

$$
f(z)-\pi_{n, m}(z ; f)=\sum_{n+m+1}^{\infty} f_{k} z^{k} .
$$

В частности, $\pi_{n, 0}(z ; f)$ есть $n$-я частная сумма ряда (1). Для мероморфных функций $f$ качественные вопросы сходимости $\pi_{n, m}(z ; f)$ к $f(z)$ при $n \rightarrow \infty$ и фиксированном $m$ детально проработаны (см., например, обзор [1]). К настоящему времени имеется уже достаточно много примеров целых функций $f$, для которых исследована сходимость аппроксимаций Паде и, в частности, найдена асимптотика убывания $f(z)-\pi_{n, m}(z ; f)$ для $z \in D$ при фиксированном $m$ и $n \rightarrow \infty$ (см. [2]-[15]).

В данной работе аналогичные исследования предприняты для функций, имеющих логарифмические точки ветвления. В частности, установлена асимптотика поведения строк таблицы Паде для функций из классов $\mathscr{A}(b), \mathscr{A}^{-}(b)$ (см. теоремы 3 и 4 ) и найдены точные порядки наилучших равномерных рациональных приближений таких функций в круге $D_{q}=\{z:|z| \leqslant q<1\}$ (см. п. 4).

2. Основные теоремы. Для $f \in \mathscr{A}(b)$ введем в рассмотрение определители Адамара ${ }^{1}$ :

$$
D_{n, m}=\left|\begin{array}{cccc}
\frac{1}{a_{n-m+1}} & \frac{1}{a_{n-m+2}} & \ldots & \frac{1}{a_{n}} \\
\frac{1}{a_{n-m+2}} & \frac{1}{a_{n-m+3}} & \ldots & \frac{1}{a_{n+1}} \\
\vdots & \vdots & \vdots & \vdots \\
\frac{1}{a_{n}} & \frac{1}{a_{n+1}} & \ldots & \frac{1}{a_{n+m-1}}
\end{array}\right| .
$$

Многочлены Паде $p_{n}(z ; f)$ и $q_{m}(z ; f)$ находятся с точностью до постоянного множителя. Будем называть стандартной такую их нормировку, при которой $q_{m}(0 ; f)=$ $D_{n, m}$. Рассмотрим также определители

$$
D_{n, m, k}=\left|\begin{array}{ccccc}
\frac{1}{a_{n-m+1}} & \frac{1}{a_{n-m+2}} & \ldots & \frac{1}{a_{n}} & \frac{1}{a_{n+1}} \\
\frac{1}{a_{n-m+2}} & \frac{1}{a_{n-m+3}} & \ldots & \frac{1}{a_{n+1}} & \frac{1}{a_{n+2}} \\
\vdots & \vdots & \vdots & \vdots & \vdots \\
\frac{1}{a_{n}} & \frac{1}{a_{n+1}} & \ldots & \frac{1}{a_{n+m-1}} & \frac{1}{a_{n+m}} \\
\frac{1}{a_{n+k}} & \frac{1}{a_{n+k+1}} & \ldots & \frac{1}{a_{n+m+k-1}} & \frac{1}{a_{n+m+k}}
\end{array}\right| .
$$

\footnotetext{
${ }^{1}$ Оператор Паде является однородным, т.е. $\pi_{n, m}(\cdot ; \lambda f)=\lambda \pi_{n, m}(\cdot ; f)$. Поэтому без ограничения общности в дальнейшем рассматриваем случай, когда перед суммой в (1) стоит знак “+”.
} 
Лемма 1. Пусть $D\left(x_{1}, x_{2}, \ldots, x_{m}\right)=\prod_{1 \leqslant i<j \leqslant m}\left(x_{i}-x_{j}\right)$ - определитель Вандермонда, $m \geqslant 2$. Тогда

$$
\operatorname{det}\left|\frac{1}{1-x_{i} y_{j}}\right|_{i, j=1}^{m}=D\left(x_{1}, x_{2}, \ldots, x_{m}\right) D\left(y_{1}, y_{2}, \ldots, y_{m}\right) \prod_{i=1}^{m} \prod_{j=1}^{m} \frac{1}{1-x_{i} y_{j}} .
$$

Данное утверждение известно как лемма Коши [16; лемма VII.6.A].

ЛЕмма 2. При $m \geqslant 2$

$$
\operatorname{det}\left|\frac{1}{x_{i}+y_{j}}\right|_{i, j=1}^{m}=D\left(x_{1}, x_{2}, \ldots, x_{m}\right) D\left(y_{1}, y_{2}, \ldots, y_{m}\right) \prod_{i=1}^{m} \prod_{j=1}^{m} \frac{1}{x_{i}+y_{j}} .
$$

ДоказАтельство. Заменив в равенстве Коши $x_{1}, x_{2}, \ldots, x_{m}$ на $-x_{1}^{-1}, x_{2}^{-1}, \ldots$, $x_{m}^{-1}$, после несложных преобразований получим нужное равенство. Лемма 2 доказана.

ЛЕмма 3. Если $f \in \mathscr{A}(b)$, то для $m \geqslant 2$

$$
D_{n, m}=b^{m(m-1)} \prod_{j=1}^{m-1}(j !)^{2} \prod_{i=1}^{m} \prod_{j=1}^{m} \frac{1}{x_{i}+y_{j}},
$$

npu $m \geqslant 1$

$$
D_{n, m, k}=b^{m(m+1)} \prod_{j=1}^{m} j ! \prod_{j=1}^{m-1} j ! \prod_{j=1}^{m}(m+k-j) \prod_{i=1}^{m+1} \prod_{j=1}^{m+1} \frac{1}{x_{i}+y_{j}},
$$

где $x_{i}=b(n-m+i)+a_{0}, i=1,2, \ldots, m, x_{m+1}=b(n+k)+a_{0}, y_{j}=b(j-1)$, $j=1,2, \ldots, m+1$.

ДоказАтельство. Из леммы 2 следует, что

$$
\begin{aligned}
D_{n, m} & =D\left(x_{1}, x_{2}, \ldots, x_{m}\right) D\left(y_{1}, y_{2}, \ldots, y_{m}\right) \prod_{i=1}^{m} \prod_{j=1}^{m} \frac{1}{x_{i}+y_{j}} \\
& =(-1)^{m(m-1)} \prod_{1 \leqslant i<j \leqslant m}\left(x_{j}-x_{i}\right) \prod_{1 \leqslant i<j \leqslant m}\left(y_{j}-y_{i}\right) \prod_{i=1}^{m} \prod_{j=1}^{m} \frac{1}{x_{i}+y_{j}} \\
& =b^{m(m-1)} \prod_{j=1}^{m-1}(j !)^{2} \prod_{i=1}^{m} \prod_{j=1}^{m} \frac{1}{x_{i}+y_{j}} .
\end{aligned}
$$

Равенство (3) доказано. Аналогично, с учетом леммы 2, получим

$$
\begin{aligned}
D_{n, m, k} & =D\left(x_{m+1}, x_{m}, \ldots, x_{1}\right) D\left(y_{m+1}, y_{m}, \ldots, y_{1}\right) \prod_{i=1}^{m+1} \prod_{j=1}^{m+1} \frac{1}{x_{i}+y_{j}} \\
& =b^{m(m+1)} \prod_{j=1}^{m} j ! \prod_{j=1}^{m-1} j ! \prod_{j=1}^{m}(m+k-j) \prod_{i=1}^{m+1} \prod_{j=1}^{m+1} \frac{1}{x_{i}+y_{j}} .
\end{aligned}
$$

Равенство (4) доказано. 
ЗАмечание 1 . По определению полагаем $D_{n, 0}=1, D_{n, 1}=1 / a_{n}, D_{n, 0, k}=1 / a_{n+k}$.

Очевидно, что $D_{n+1, m+1}=D_{n, m, 1}$. Поэтому из (3) следует, что

$$
\frac{D_{n, m, 1}}{D_{n, m}}=b^{2 m}(m !)^{2} \prod_{i=1}^{m} \frac{1}{x_{i}+y_{m+1}} \prod_{j=1}^{m+1} \frac{1}{x_{m+1}+y_{j}}=b^{2 m}(m !)^{2} \frac{1}{a_{n+m+1}} \prod_{i=1}^{m} \frac{1}{a_{n+i}^{2}} .
$$

Рассмотрим аналитическую в $D$ функцию $[17 ;$ гл. $5, \S 2.3,(1)]$

$$
\varphi(z)=\sum_{k=0}^{\infty} \frac{z^{k}}{(k+n+1)^{m+1}}=\frac{1}{m !} \int_{0}^{\infty} \frac{t^{m} e^{-(n+1) t} d t}{1-z e^{-t}} .
$$

Нетрудно показать, что если $z \in D$

$$
\varphi^{(m)}(z)=\int_{0}^{\infty} \frac{t^{m} e^{-(n+m+1) t} d t}{\left(1-z e^{-t}\right)^{m+1}}=\sum_{k=1}^{\infty} k(k+1) \cdots(k+m-1) \frac{z^{k-1}}{(k+n+m)^{m+1}} .
$$

При $z \in D$ полагаем по определению

$$
\begin{aligned}
\psi_{n, m}(z) & :=\frac{(n+m+1)^{m+1}}{m !} \varphi^{(m)}(z) \\
& =\sum_{k=1}^{\infty} \frac{k(k+1) \cdots(k+m-1)}{m !}\left(\frac{n+m+1}{n+m+k}\right)^{m+1} z^{k-1} \\
& =\frac{(n+m+1)^{m+1}}{m !} \int_{0}^{\infty} \frac{t^{m} e^{-(n+m+1) t} d t}{\left(1-z e^{-t}\right)^{m+1}}
\end{aligned}
$$

Так как

$$
\int_{0}^{\infty} t^{m} e^{-(n+m+1) t} d t=\frac{m !}{(n+m+1)^{m+1}},
$$

из (6) получаем, что для $z \in D$

$$
\frac{1}{(1+|z|)^{m+1}} \leqslant\left|\psi_{n, m}(z)\right| \leqslant \frac{1}{(1-|z|)^{m+1}} .
$$

Теорема 1. Если $f \in \mathscr{A}(b)$, то для любого фиксированного $m \in \mathbb{N} \cup\{0\}$ локально равномерно по $z \in D$ при $n \rightarrow \infty$

$$
q_{m}(z ; f) f(z)-p_{n}(z, f)=D_{n, m, 1} \psi_{n, m}(z) z^{n+m+1}(1+o(1)) .
$$

ДокАЗАтЕЛЬСтво. Согласно теореме Паде [18; гл. 1, § 1.1, (1.11)] при выбранной нормировке многочленов Паде

$$
q_{m}(z ; f) f(z)-p_{n}(z, f)=\sum_{k=1}^{\infty} D_{n, m, k} z^{n+m+k}=D_{n, m, 1} z^{n+m+1} \sum_{k=1}^{\infty} \frac{D_{n, m, k}}{D_{n, m, 1}} z^{k-1} .
$$

Из равенств (4) следует, что

$$
\frac{D_{n, m, k}}{D_{n, m, 1}}=\frac{\prod_{j=1}^{m}(m+k-j)}{m !} \prod_{j=1}^{m+1} \frac{b(n+j)+a_{0}}{b(n+k+j-1)+a_{0}} .
$$


Тогда, полагая $\tau_{0}=a_{0} / b$ и принимая во внимание (4), получим, что

$$
\psi_{n, m}(z)-\sum_{k=1}^{\infty} \frac{D_{n, m, k}}{D_{n, m, 1}} z^{k-1}=\sum_{k=1}^{\infty} \frac{k(k+1) \cdots(k+m-1)}{m !} \alpha_{n, m, k} z^{k-1},
$$

где

$$
\alpha_{n, m, k}=\left(\frac{n+m+1}{n+m+k}\right)^{m+1}-\prod_{j=1}^{m+1} \frac{n+j+\tau_{0}}{n+j+k-1+\tau_{0}} .
$$

В случае, если $\alpha_{n, m, k} \geqslant 0$, будем иметь

$$
0 \leqslant \alpha_{n, m, k} \leqslant\left(\frac{n+m+1}{n+m+k}\right)^{m+1}-\left(\frac{n+1+\tau_{0}}{n+k+\tau_{0}}\right)^{m+1} \leqslant \frac{m(m+1)(k-1)}{(n+m+k)(n+k)} .
$$

Если же $\alpha_{n, m, k}<0$, то

$$
0 \leqslant\left|\alpha_{n, m, k}\right| \leqslant\left(\frac{n+m+1+\tau_{0}}{n+m+k+\tau_{0}}\right)^{m+1}-\left(\frac{n+m+1}{n+m+k}\right)^{m+1} \leqslant \frac{(m+1) \tau_{0}(k-1)}{(n+m+k)(n+k)} .
$$

Следовательно,

$$
\left|\alpha_{n, m, k}\right| \leqslant \frac{(m+1)\left(m+\tau_{0}\right)}{n} \leqslant c \frac{m^{2}}{n},
$$

где $c$ - положительная постоянная. Отсюда, из (7), (9) и легко доказываемого неравенства

следует, что

$$
\frac{k(k+1) \cdots(k+m-1)}{m !} \leqslant k^{m}
$$

$$
\sum_{k=1}^{\infty} \frac{D_{n, m, k}}{D_{n, m, 1}} z^{k-1}=\psi_{n, m}(z)\left(1+\beta_{n, m, k}(z)\right)
$$

где

$$
\left|\beta_{n, m, k}(z)\right| \leqslant c \frac{2^{m+1} m^{2}}{n} \sum_{k=1}^{\infty} k^{m}|z|^{k-1} .
$$

Из равенства (см. [17; гл. $5, \S 2.2,(3)])$

$$
\sum_{k=1}^{\infty} k^{m}|z|^{k-1}=\sum_{k=1}^{m} \frac{(-1)^{k}|z|^{k-1}}{(1-|z|)^{k+1}}\left[\sum_{j=1}^{k}(-1)^{j} C_{j}^{k} j^{m}\right],
$$

где $C_{j}^{k}$ - биномиальные коэффициенты, следует, что при $z \in D$

$$
\sum_{k=1}^{\infty} k^{m}|z|^{k-1} \leqslant \frac{2^{m} m^{m+1}}{(1-|z|)^{m+1}} .
$$

Поэтому справедливо неравенство

$$
\left|\beta_{n, m, k}(z)\right| \leqslant c \frac{2^{2 m+1} m^{m+3}}{n(1-|z|)^{m+1}} .
$$

Из (10) при фиксированном $m$ и $n \rightarrow \infty$ получаем, что $\beta_{n, m, k}(z)=o(1)$ для каждого фиксированного $z \in D$. Теорема 1 доказана. 
ЗАмечАниЕ 2. Из доказательства теоремы 1 следует, что (8) справедливо при $n \rightarrow \infty$ и в том случае, когда $m \rightarrow \infty$ достаточно медленно, в частности, если $m=O(\ln (\ln n)) .^{2}$

СлеДСтвиЕ 1. Если $f \in \mathscr{A}^{-}(b)$, то для любого фиксированного $m \in \mathbb{N} \cup\{0\}$ локально равномерно по $z \in D$ при $n \rightarrow \infty$

$$
q_{m}(z ; f) f(z)-p_{n}(z, f)=D_{n, m, 1} \psi_{n, m}(-z)(-z)^{n+m+1}(1+o(1)) .
$$

Теорема 2. Если $f \in \mathscr{A}(b)$, то для любого фиксированного $m \in \mathbb{N} \cup\{0\}$ локально равномерно по $z \in D$ при $n \rightarrow \infty$

$$
q_{m}(z ; f)=D_{n, m}(1-z)^{m}(1+o(1)) .
$$

ДокАЗАТЕЛЬСтво. Представим многочлен $q_{m}(z ; f)$ в виде

$$
q_{m}(z ; f)=\sum_{j=0}^{m} q_{j} z^{j} .
$$

Тогда согласно равенству (1.8) из [18] получим (столбец выделенный полужирным шрифтом следует опустить)

$$
(-1)^{j} q_{j}=\left|\begin{array}{ccccccc}
\frac{1}{a_{n-m+1}} & \cdots & \frac{1}{a_{n-j}} & \frac{\mathbf{1}}{\boldsymbol{a}_{\boldsymbol{n}-\boldsymbol{j}+\mathbf{1}}} & \frac{1}{a_{n-j+2}} & \ldots & \frac{1}{a_{n+1}} \\
\frac{1}{a_{n-m+2}} & \cdots & \frac{1}{a_{n-j+1}} & \frac{\mathbf{1}}{\boldsymbol{a}_{\boldsymbol{n}-\boldsymbol{j}+\mathbf{2}}} & \frac{1}{a_{n-j+3}} & \ldots & \frac{1}{a_{n+2}} \\
\vdots & \vdots & \vdots & \vdots & \vdots & \vdots & \vdots \\
\frac{1}{a_{n-1}} & \cdots & \frac{1}{a_{n-j+m-2}} & \frac{\mathbf{1}}{\boldsymbol{a}_{\boldsymbol{n}-\boldsymbol{j}+\boldsymbol{m}-\mathbf{1}}} & \frac{1}{a_{n-j+m}} & \cdots & \frac{1}{a_{n+m-1}} \\
\frac{1}{a_{n}} & \cdots & \frac{1}{a_{n-j+m-1}} & \frac{1}{\boldsymbol{a}_{\boldsymbol{n}-\boldsymbol{j}+\boldsymbol{m}}} & \frac{1}{a_{n-j+m+1}} & \cdots & \frac{1}{a_{n+m}}
\end{array}\right| .
$$

Полагая, как и раньше, $x_{i}=b(n-m+i)+a_{0}, i=1,2, \ldots, m$, a

$$
y_{k}^{*}= \begin{cases}b(k-1), & k=1,2, \ldots, m-j, \\ b k, & k=m-j+1, \ldots, m,\end{cases}
$$

и, применяя к последнему определителю лемму 2, с учетом равенства (3) будем иметь

$$
\begin{aligned}
(-1)^{j} q_{j} & =b^{m(m-1) / 2} \prod_{p=1}^{m-1} p ! \prod_{1 \leqslant i<j \leqslant m}\left(y_{j}^{*}-y_{i}\right) \prod_{i=1}^{m} \prod_{p=1}^{m} \frac{1}{x_{i}+y_{p}^{*}} \\
& =\frac{b^{m(m-1)}}{(m-j) ! j !} \prod_{p=1}^{m-1} p ! \prod_{p=1}^{m} p ! \prod_{i=1}^{m} \prod_{p=1}^{m} \frac{1}{x_{i}+y_{p}^{*}}
\end{aligned}
$$

\footnotetext{
${ }^{2}$ Можно показать, что все доказанные далее теоремы верны при указанных в замечании ограничениях на рост $m$. При $m \rightarrow \infty$ формулировку теоремы 1 , как и формулировки последующих теорем, нужно подправить, рассматривая в них вместо фиксированного $m$, например, произвольную последовательность $m=m(n)=O(\ln (\ln n))$.
} 


$$
\begin{aligned}
& =b^{m(m-1)} C_{j}^{m} \prod_{p=1}^{m-1}(p !)^{2} \prod_{i=1}^{m} \prod_{p=1}^{m} \frac{1}{x_{i}+y_{p}} \prod_{i=1}^{m} \prod_{p=m-j+1}^{m} \frac{x_{i}+y_{p}}{x_{i}+y_{p}^{*}} \\
& =D_{n, m} C_{j}^{m} \prod_{i=1}^{m} \prod_{p=m-j+1}^{m} \frac{x_{i}+y_{p}}{x_{i}+y_{p}^{*}},
\end{aligned}
$$

где $C_{j}^{m}=m ! /((m-j) ! j !)$ - биномиальные коэффициенты. Тогда для любого $z \in D$

$$
D_{n, m}(1-z)^{m}-q_{m}(z ; f)=D_{n, m} \sum_{j=0}^{m} C_{j}^{m}(-z)^{j}\left\{1-\prod_{i=1}^{m} \prod_{p=m-j+1}^{m} \frac{x_{i}+y_{p}}{x_{i}+y_{p}^{*}}\right\} .
$$

Поскольку

$$
\begin{aligned}
1-\prod_{i=1}^{m} \prod_{p=m-j+1}^{m} \frac{x_{i}+y_{p}}{x_{i}+y_{p}^{*}} & \leqslant 1-\prod_{i=1}^{m}\left(\frac{x_{i}+y_{m-j+1}}{x_{i}+y_{m-j+1}^{*}}\right)^{j} \leqslant 1-\left(\frac{x_{1}+b(m-j)}{x_{1}+b(m-j+1)}\right)^{m j} \\
& \leqslant 1-\left(\frac{x_{1}}{x_{1}+b}\right)^{m^{2}} \leqslant m^{2}\left(1-\frac{x_{1}}{x_{1}+b}\right) \leqslant \frac{m^{2}}{n-m+1}
\end{aligned}
$$

окончательно будем иметь

$$
\left|D_{n, m}(1-z)^{m}-q_{m}(z ; f)\right|=D_{n, m} \frac{m^{2}}{n-m+1} \sum_{j=0}^{m} C_{j}^{m} q^{j} \leqslant \frac{m^{2}(1+q)^{m}}{n-m+1} D_{n, m} .
$$

Отсюда для любого $z \in D$

$$
q_{m}(z ; f)=D_{n, m}(1-z)^{m}\left(1+\beta_{n, m}(z)\right),
$$

где

$$
\left|\beta_{n, m}(z)\right| \leqslant \frac{m^{2}(1+q)^{m}}{(n-m+1)(1-|z|)^{m}} .
$$

Из последних двух соотношений и следует утверждение теоремы. Теорема 2 доказана.

ЗАмечаниЕ 3. Из теоремы 2 следует, что при фиксированном $m$ и $n \rightarrow \infty$ все полюсы дроби Паде $\pi_{n, m}(z ; f)$ притягиваются особой точкой $z_{0}=1$ функции $f$, которая является ее логарифмической точкой ветвления.

СлЕДСтвиЕ 2. Если $f \in \mathscr{A}^{-}(b)$, то для любого фиксированного $m \in \mathbb{N} \cup\{0\}$ локально равномерно по $z \in D$ при $n \rightarrow \infty$

$$
q_{m}(z ; f)=D_{n, m}(1+z)^{m}(1+o(1)) .
$$

ТеОрема 3. Если $f \in \mathscr{A}(b)$, то для любого фиксированного $m \in \mathbb{N} \cup\{0\}$ локально равномерно по $z \in D$ при $n \rightarrow \infty$

$$
\begin{aligned}
f(z)-\pi_{n, m}(z ; f) & =\frac{D_{n, m, 1}}{D_{n, m}} \frac{\psi_{n, m}(z)}{(1-z)^{m}} z^{n+m+1}(1+o(1)) \\
& =\frac{(m !)^{2}}{b n^{2 m+1}} \frac{\psi_{n, m}(z)}{(1-z)^{m}} z^{n+m+1}(1+o(1)) .
\end{aligned}
$$


ДокАЗАТЕЛЬство. Утверждение теоремы непосредственно следует из теорем 1 , 2 и равенства (5).

Теорема 4. Если $f \in \mathscr{A}^{-}(b)$, то для любого фиксированного $m \in \mathbb{N} \cup\{0\}$ локально равномерно по $z \in D$ при $n \rightarrow \infty$

$$
f(z)-\pi_{n, m}(z ; f)=\frac{(m !)^{2}}{b n^{2 m+1}} \frac{\psi_{n, m}(-z)}{(1+z)^{m}}(-z)^{n+m+1}(1+o(1)) .
$$

3. Некоторые обобщения. Пусть $g \in \mathscr{A}(b ; d)$, т.е.

$$
g(z)= \pm \sum_{n=0}^{\infty} \frac{z^{l_{n}}}{a_{n}}=z^{l_{0}} f\left(z^{d}\right)
$$

где $f \in \mathscr{A}(b)$. Обозначим через $\pi_{n, m}(\xi ; f)$ соответствующую аппроксимацию Паде функции $f(\xi)$. Она существует, так как из равенства (3) следует, что $D_{n, m} \neq 0$. Следовательно,

$$
f(\xi)-\pi_{n, m}(\xi ; f)=f_{n+m+1} \xi^{n+m+1}+\cdots .
$$

Полагая в последнем равенстве $\xi=z^{d}$, а затем умножая его на $z^{l_{0}}$, получим

$$
z^{l_{0}} f\left(z^{d}\right)-z^{l_{0}} \pi_{n, m}\left(z^{d} ; f\right)=f_{n+m+1} z^{d(n+m+1)+l_{0}}+\cdots .
$$

Из последнего равенства следует, что

$$
\pi_{l_{n}+i, d m+j}(z ; g)=z^{l_{0}} \pi_{n, m}\left(z^{d} ; f\right)
$$

где $0 \leqslant i+j \leqslant d-1 .^{3}$

Используя равенство (13), легко получить аналоги теорем (3) и (4) для классов $\mathscr{A}^{ \pm}(b ; d)$.

Теорема 5. Пусть $g \in \mathscr{A}(b ; d)$. Тогда для любого фиксированного $m \in \mathbb{N} \cup\{0\}$ локально равномерно по $z \in D$ при $n \rightarrow \infty$

$$
\begin{aligned}
g(z)-\pi_{l_{n}+i, d m+j}(z ; g) & =\frac{D_{n, m, 1}}{D_{n, m}} \frac{\psi_{n, m}\left(z^{d}\right)}{\left(1-z^{d}\right)^{m}} z^{l_{n}+d m+d}(1+o(1)) \\
& =\frac{(m !)^{2}}{b n^{2 m+1}} \frac{\psi_{n, m}\left(z^{d}\right)}{\left(1-z^{d}\right)^{m}} z^{l_{n}+d m+d}(1+o(1)),
\end{aligned}
$$

где $0 \leqslant i+j \leqslant d-1$.

Tеорема 6. Пусть $g \in \mathscr{A}^{-}(b ; d)$. Тогда для любого фиксированного $m \in \mathbb{N} \cup\{0\}$ локально равномерно по $z \in D$ при $n \rightarrow \infty$

$$
g(z)-\pi_{l_{n}+i, d m+j}(z ; g)=\frac{(-1)^{n+m+1}(m !)^{2}}{b n^{2 m+1}} \frac{\psi_{n, m}\left(-z^{d}\right)}{\left(1+z^{d}\right)^{m}} z^{l_{n}+d m+d}(1+o(1)),
$$

где $0 \leqslant i+j \leqslant d-1$.

\footnotetext{
${ }^{3} \mathrm{C}$ помощью теоремы Паде-Бейкера о блочной структуре таблицы Паде [18; гл. $1, \S 1.4$, теорема 1.4.3] можно показать, что в оставшемся нерассмотренным случае, когда $i+j=d$ и $i, j \neq d$, аппроксимации Паде $\pi_{l_{n}+i, d m+j}(z ; g)$ в смысле принятого нами определения по Бейкеру [18; гл. 1, $\S 1.4,(4.6)]$ не существуют. Классические аппроксимации Паде $\widetilde{\pi}_{l_{n}+i, d m+j}(z ; g)$ существуют и в этом случае совпадают с $\pi_{l_{n}, d m}(z ; g)$.
} 
СлеДСтвие 3. Если $f \in \mathscr{A}^{ \pm}(b)$, то локалъно равномерно по $z \in D$ nрu $n \rightarrow \infty$

$$
f(z)-\pi_{n, 0}(z ; f)=\frac{\psi_{n, 0}( \pm z)}{b n}( \pm z)^{n+1}(1+o(1))
$$

¿əe

$$
\psi_{n, 0}(z)=\sum_{k=0}^{\infty} \frac{n+1}{k+n+1} z^{k}=(n+1) \int_{0}^{\infty} \frac{e^{-(n+1) t}}{1-z e^{-t}} d t .
$$

Аналоги следствия 3 справедливы и для функций из классов $\mathscr{A}(b ; d), \mathscr{A}^{-}(b ; d)$. Из теорем 3 и 6 , в частности, получим, что для любого фиксированного $m \in \mathbb{N} \cup\{0\}$ локально равномерно по $z \in D$ при $n \rightarrow \infty$

$$
\ln (1-z)-\pi_{n, m}(z ; \ln (1-\xi))=-\frac{(m !)^{2}}{n^{2 m+1}} \frac{\psi_{n, m}(z)}{(1-z)^{m}} z^{n+m+1}(1+o(1)),
$$

$\operatorname{arctg} z-\pi_{2 n+1+i, 2 m+j}(z ; \operatorname{arctg} \xi)=(-1)^{n+m+1} \frac{(m !)^{2}}{2 n^{2 m+1}} \frac{\psi_{n, m}\left(-z^{2}\right)}{\left(1+z^{2}\right)^{m}} z^{2 n+2 m+3}(1+o(1))$,

где $0 \leqslant i+j \leqslant 1$.

4. Наилучшие приближения функций из $\mathscr{A}^{ \pm}(b ; d)$. Определим наилучшие равномерные рациональные приближения функции $f$, аналитической в круге $D_{q}=$ $\{z:|z| \leqslant q<1\}:$

$$
R_{n, m}(f)=R_{n, m}\left(f ; D_{q}\right)=\inf \left\{\|f-r\|_{q}: r \in \mathscr{R}_{n, m}\right\},
$$

где $\|g\|_{q}=\sup \left\{|g(z)|: z \in D_{q}\right\}$, а $\mathscr{R}_{n, m}-$ множество всех рациональных функций, представимых в виде (2). Полученные в предыдущих разделах результаты позволяют получить точные порядковые оценки для $R_{n, m}\left(f ; D_{q}\right)$ при $n \rightarrow \infty$ в случае, когда функция $f$ принадлежит одному из рассматриваемых классов.

СлЕДСтвиЕ 4. Если функиия $f \in \mathscr{A}^{ \pm}(b)$, то при любом фиксированном $m \in$ $\mathbb{N} \cup\{0\}$ u $n \rightarrow \infty$

$$
\frac{(m !)^{2}}{n^{2 m+1}} \frac{q^{n+m+1}}{(1+q)^{2 m+1}}\left(1-o_{1}(1)\right) \leqslant R_{n, m}\left(f ; D_{q}\right) \leqslant \frac{(m !)^{2}}{n^{2 m+1}} \frac{q^{n+m+1}}{(1-q)^{2 m+1}}\left(1+o_{2}(1)\right),
$$

m.e.

$$
R_{n, m}\left(f ; D_{q}\right) \asymp \frac{q^{n}}{n^{2 m+1}} \asymp \frac{1}{n^{2 m}} R_{n, 0}\left(f ; D_{q}\right) .
$$

Здесь и далее бесконечно малые (б.м.) при $n \rightarrow \infty$ величины $o_{i}(1) \geqslant 0, i=1,2$, а соотношение $\alpha_{n} \asymp \beta_{n}$ означает, что б.м. $\left\{\alpha_{n}\right\},\left\{\beta_{n}\right\}$ имеют одинаковый порядок, т.е. существуют такие положительные постоянные $A$ и $B$, что $A \alpha_{n} \leqslant \beta_{n} \leqslant B \alpha_{n}$ при $n=0,1,2, \ldots$.

Доказательство. Рассмотрим функцию $\varphi(z):=f(z)-\pi_{n, m}(z ; f)$. Из теорем 3,4 следует, что при достаточно больших $n \varphi$ является аналитической внутри круга $D_{q_{1}}$, $0<q<q_{1}<1$ и имеет в $D_{q}$ нуль кратности $n+m+1$. Покажем, что

$$
\min _{|z|=q}|\varphi(z)| \leqslant R_{n, m}\left(f ; D_{q}\right) \leqslant \max _{|z|=q}|\varphi(z)| .
$$

Поскольку правое неравенство в (15) вполне очевидно, остановимся на доказательстве только левого неравенства. Для этого нам необходима следующая лемма Гончара-Дзядыка [19; лемма 3.1]. 
ЛЕмма 4. Если аналитическая в односвязной области $G$ и непрерьвная на $\bar{G}$ функиия $\varphi$ имеет в $G$ с учетом кратности по крайней мере $n+1$ нуль, то при произвольном $m \geqslant 0$ справедливо неравенство

$$
R_{n, m}(\varphi ; \bar{G}) \geqslant \min _{z \in \partial G}|\varphi(z)| .
$$

Пусть $r_{n, m}^{*} \in \mathscr{R}_{n, m}$ и является рациональной функцией наилучшего равномерного приближения $f$ в круге $D_{q}$. Тогда, применяя к функции $\varphi$ лемму 4 , получим

$$
\begin{aligned}
R_{n, m}\left(f ; D_{q}\right) & =\left\|f-r_{n, m}^{*}\right\|=\left\|f-\pi_{n, m}-\left(r_{n, m}^{*}-\pi_{n, m}\right)\right\| \\
& =\left\|\varphi-\widetilde{r}_{n+m, 2 m}\right\| \geqslant R_{n+m, 2 m}\left(\varphi ; D_{q}\right) \geqslant \min _{|z|=q}|\varphi(z)| .
\end{aligned}
$$

Неравенство (15) доказано. Из (15), а также из равенств (11), (12) и неравенства (7) следует (14). Следствие 4 доказано.

Аналогично доказывается

СлеДСтвиЕ 5. Если $g \in \mathscr{A}^{ \pm}(b ; d)$, то при любом фиксированном $m \in \mathbb{N} \cup\{0\} u$ $n \rightarrow \infty$

$\frac{(m !)^{2}}{b n^{2 m+1}} \frac{q^{l_{n}+d m+d}}{\left(1+q^{d}\right)^{2 m+1}}\left(1-o_{1}(1)\right) \leqslant R_{l_{n}+i, d m+j}\left(g ; D_{q}\right) \leqslant \frac{(m !)^{2}}{b n^{2 m+1}} \frac{q^{l_{n}+d m+d}}{\left(1-q^{d}\right)^{2 m+1}}\left(1+o_{2}(1)\right)$,

m.e.

$$
R_{l_{n}+i, d m+j}\left(g ; D_{q}\right) \asymp \frac{q^{d n}}{n^{2 m+1}} \asymp \frac{1}{n^{2 m}} R_{l_{n}+i, 0}\left(g ; D_{q}\right),
$$

где $0 \leqslant i+j \leqslant d-1$.

В частности, из следствий 4,5 получим, что при фиксированном $m \in \mathbb{N} \cup\{0\}$ и $n \rightarrow \infty$

$$
\begin{aligned}
R_{n, m}\left(\ln (1-z) ; D_{q}\right) & \asymp \frac{q^{n}}{n^{2 m+1}} \asymp \frac{1}{n^{2 m}} R_{n, 0}\left(\ln (1-z) ; D_{q}\right), \\
R_{2 n+1+i, 2 m+j}\left(\operatorname{arctg} z ; D_{q}\right) & \asymp \frac{q^{2 n}}{n^{2 m+1}} \asymp \frac{1}{n^{2 m}} R_{2 n+1+i, 0}\left(\operatorname{arctg} z ; D_{q}\right),
\end{aligned}
$$

где $0 \leqslant i+j \leqslant 1$.

Основные результаты работы частично анонсированы в [20].

\section{СПИСОК ЦИТИРОВАННОЙ ЛИТЕРАТУРЫ}

[1] С. П. Суетин, “Аппроксимации Паде и эффективное аналитическое продолжение степенного ряда", УМН, 57:1 (2002), 45-142.

[2] E. B. Saff, "The convergence of rational functions of best approximation to the exponential function. II", Proc. Amer. Math. Soc., 32:1 (1972), 187-194.

[3] E. B. Saff, "On the degree of best rational approximation to the exponential function", J. Approximation Theory, 9:2 (1973), 97-101.

[4] D. Braess, "On the conjecture of Meinardus on rational approximation of $e^{x}$ ", J. Approximation Theory, 40:4 (1984), 375-379.

[5] D. S. Lubinsky, "Padé tables of entire functions of very slow and smooth growth", Constr. Approx., 1:4 (1985), 349-358. 
[6] D. S. Lubinsky, "Uniform convergence of rows of the Padé table for functions with smooth Maclaurin series coefficients", Constr. Approx., 3:3 (1987), 307-330.

[7] D. S. Lubinsky, "Padé tables of entire functions of very slow and smooth growth. II", Constr. Approx., 4:3 (1988), 321-339.

[8] A.L. Levin, D. S. Lubinsky, "Best rational approximation of entire functions whose Maclaurin series coefficients decrease rapidly and smoothly", Trans. Amer. Math. Soc., 293:2 (1986), 533-545.

[9] A. L. Levin, D. S. Lubinsky, "Rows and diagonals of the Walsh array for entire functions with smooth Maclaurin series coefficients", Constr. Approx., 6:3 (1990), 257-286.

[10] Л. Л. Березкина, В.Н.Русак, "О наилучших рациональных аппроксимациях некоторых целых функций”, Весці АН БССР. Сер. фіз.-матэм. навук, 1990, № 4, 27-32.

[11] В.Н. Русак, Та Хонг Куанг, “Асимптотика параболических звеньев рациональной таблицы Чебышева для аналитических функций”, Докл. АН БССР, 34:10 (1990), 869-871.

[12] А. П. Старовойтов, Н. А. Старовойтова, “Асимптотика определителей Адамара и поведение строк таблиц Паде и Чебышева для суммы экспонент", Матем. сб., 187:2 (1996), 141-157.

[13] В.Н. Русак, А. П. Старовойтов, “Аппроксимации Паде для целых функций с регулярно убывающими коэффициентами Тейлора", Матем. сб., 193:9 (2002), 63-92.

[14] А. П. Старовойтов, Н. А. Старовойтова, "Аппроксимации Паде одного класса целых функций”, Докл. НАН Беларуси, 50:6 (2006), 28-30.

[15] А. П. Старовойтов, Н. А. Старовойтова, “Аппроксимации Паде функций Миттаг-Леффлера", Матем. сб., 198:7 (2007), 109-122.

[16] Г. Вейль, Классические группы: их инварианты и представления, ИЛ, М., 1947.

[17] А. П. Прудников, Ю.А. Брычков, О. И. Маричев, Интеграль и ряды, Элементарные функции, Наука, М., 1981.

[18] Дж. Бейкер, П. Грейвс-Моррис, Аппроксимации Паде, 1. Основы теории; 2. Обобщения и приложения, Мир, М., 1986.

[19] В.К. Дзядык, “Об асимптотике диагональных аппроксимаций Паде функций $\sin z$, $\cos z, \operatorname{sh} z$ и $\operatorname{ch} z "$, Матем. сб., 108:2 (1979), 247-267.

[20] А. П. Старовойтов, Н. А. Старовойтова, "О приближении рациональными операторами Паде”, Еругинские чтения XI: Тез. докл. междунар. конф. (24-26 мая 2006 г., Гомель, Беларусь), Ин-т матем. НАН Беларуси, Гомель, 2006, 127-128.

\section{А. П. Старовойтов}

Поступило

Гомельский государственный университет им. Ф. Скорины

11.05.2007

E-mail: svoitov@gsu. unibel.by

\section{Н. А. Старовойтова}

Гомельский государственный университет им. Ф. Скорины

E-mail: svoitov@gsu.unibel.by 